\title{
Disparidades Intrarregionais na Região Nordeste do Brasil
}

\section{Intra-regional Disparities in the Northeast Region of Brazil}

\author{
Luiz Carlos de Santana Ribeiro* \\ Fernando Salgueiro Perobelli** \\ Edson Paulo Domingues***
}

\begin{abstract}
Resumo: O objetivo deste trabalho é investigar as disparidades intrarregionais da região Nordeste do Brasil, a partir da diferenciação da sua estrutura produtiva. Para isso, aplica-se a análise de decomposição espacial inspirada em Feldman et al. (1987) e adaptada de acordo com as propostas de Dietzenbacher et al. (2000) e Jackson e Dzikowski (2002). A base de dados utilizada é uma matriz de insumo-produto (GUILHOTO et al., 2010), ano base 2004, com 111 setores econômicos e 10 regiões (nove estados do Nordeste e restante do Brasil). Os principais resultados apontam que a demanda final desempenha papel relevante na determinação da diferença do produto das atividades ligadas ao segmento de serviços, principalmente dos estados da Bahia, Ceará e Pernambuco. Por outro lado, a demanda final não é responsável pelas diferenças no produto entre algumas atividades da agropecuária, de setores ligados à indústria de alimentos e da produção de fumo. Além disso, a demanda final não explica as diferenças na produção de praticamente todas as atividades dos estados de Alagoas, Maranhão, Paraíba, Piauí, Sergipe e Rio Grande do Norte.
\end{abstract}

Palavras-chave: Insumo-produto. Análise de decomposição espacial. Região Nordeste.

Abstract: This paper aims to investigate the intraregional inequalities in the Brazilian Northeast, based on the differentiation of its productive structure. For this, a spatial decomposition analysis is applied, inspired by Feldman et al. (1987) and adapted according to Dietzenbacher et al. (2000) and Jackson and Dzikowski (2002). An input-output matrix is used (GUILHOTO et al., 2010), base year 2004, with 111 sectors and 10 regions (9 states of the Northeast and the rest of Brazil). The main results indicate that the final demand plays a significant role in determining the difference in the sectors output related to the services segment, mainly in the states of Bahia, Ceará and Pernambuco. On the other hand, final demand was not responsible for certain differences in the output between some agricultural activities, sectors related to the food industry and tobacco. In addition, final demand

* Doutor em Economia pelo Centro de Desenvolvimento e Planejamento Regional (Cedeplar) da Universidade Federal de Minas Gerais (UFMG). Professor do Programa de Pós-Graduação em Economia da Universidade Federal de Sergipe (UFS). Coordenador do Laboratório de Economia Aplicada e Desenvolvimento Regional (Leader). Bolsista de Produtividade em Pesquisa do CNPq. E-mail: ribeiro.luiz84@gmail.com

* Doutor em Economia pela Universidade de São Paulo (USP). Professor do Programa de PósGraduação em Economia da Universidade Federal de Juiz de Fora (UFJF). Coordenador do Laboratório de Análises Territoriais e Setoriais (Lates). Bolsista de Produtividade em Pesquisa do CNPq. E-mail: fernandosalgueiro.perobelli@gmail.com

*** Doutor em Economia pela Universidade de São Paulo (USP). Professor do Programa de PósGraduação em Economia do Centro de Desenvolvimento e Planejamento Regional (Cedeplar) da Universidade Federal de Minas Gerais (UFMG). Coordenador do Núcleo de Estudos em Modelagem Econômica e Ambiental Aplicada (Nemea). Bolsista de Produtividade em Pesquisa do CNPq. E-mail: domingues.edson@gmail.com 
does not explain the output differences in almost all sectors among the states of Alagoas, Maranhão, Paraíba, Piauí, Sergipe and Rio Grande do Norte.

Keywords: Input-output. Spatial decomposition analysis. Northeastern Region.

JEL Classification: R-15.

\section{Introdução}

O papel do centro dinâmico da região nordeste capaz de impulsionar o seu crescimento foi desempenhado, inicialmente, de forma modesta pela demanda externa, o que constituiu o ciclo econômico primário-exportador. Mais tarde, esse papel coube aos investimentos em infraestrutura e aos investimentos industriais financiados pelo governo federal a fundo perdido. Entretanto, o impulso de crescimento gerado tanto pela economia primária-exportadora quanto pelo ciclo industrial não teve praticamente nenhuma relação com a dinâmica regional interna, o que refletiu na inexistência de transformações sociais, impedindo o processo de desenvolvimento da região (FURTADO, 1959).

Os investimentos industriais realizados inicialmente a partir da política desenvolvimentista da Superintendência do Desenvolvimento do Nordeste (Sudene) nos anos 1960 foram concentrados nos estados da Bahia, Ceará e Pernambuco. Isso, por sua vez, contribuiu para o aumento das disparidades e da heterogeneidade no Nordeste (LIMA, 1994; GOMES; VERGULINO, 1995; ARAÚJO, 2004, 2008), criando simultaneamente subespaços baseados em estruturas dinâmicas e modernas e áreas estagnadas (GUIMARÃES NETO, 1997; ARAÚJO, 2004) que, mesmo quando apresentam algum tipo de modernização, são bastante limitadas. Esses contrastes presentes na região deram origem à expressão "vários Nordestes", como destacado por Araújo (2004).

Nesse contexto, qual é a estrutura das disparidades regionais no Nordeste, do ponto de vista estadual? Mais especificamente, será que a diferença entre suas estruturas produtivas estaduais pode explicar parte dessa disparidade? Na tentativa de responder tais questionamentos, recorre-se aos modelos de insumo-produto.

Esses modelos refletem a estrutura econômica de determinada região por meio da representação dos fluxos monetários de bens e serviços entre seus diversos setores de atividade econômica. Em outras palavras, eles podem ser vistos como uma fotografia da economia em um determinado período de tempo. Para Miller e Blair (2009), a modelagem interregional, embora necessite de um número muito superior de dados estatísticos, é mais apropriada do que os modelos construídos para apenas uma região, pois as ligações interregionais são captadas, ou seja, aquela região de estudo não está isolada ou desconexa do restante do país no qual está inserida. 
Os modelos de insumo-produto possibilitam a melhor compreensão da interdependência entre os setores produtivos de uma economia, bem como permitem a identificação de fragilidades e potencialidades do ponto de vista intersetorial que podem contribuir para o desenvolvimento regional. Há um grande número de indicadores possíveis de se calcular a partir das tabelas de insumo-produto. Entre eles, existe a análise de decomposição estrutural (SDA), que busca captar mudanças estruturais na economia a partir da ótica intersetorial (coeficientes técnicos) ou da demanda final (JACKSON; DZIKOWSKI, 2002).

A SDA é uma técnica de estática comparativa que pode ser utilizada para decompor mudanças ocorridas em variáveis macroeconômicas de determinada economia por meio de matrizes de insumo-produto (MIPs). A referência teórica original da SDA é encontrada nos trabalhos de Chenery et al. (1962), Leontief (1966) e Carter (1970), com contribuições relevantes de Blair e Wyckoff (1989), Skolka (1989) e Rose e Casler (1996). A partir da SDA, percebe-se que, para quaisquer mudanças na produção, parte ocorre por causa de mudanças nos coeficientes técnicos e parte é decorrente de mudanças na estrutura da demanda final (CHÓLIZ; DUARTE, 2006).

Essa decomposição pode ser realizada temporalmente, a partir de pelo menos duas matrizes para anos distintos, e também espacialmente. ${ }^{1}$ Em relação a trabalhos recentes que utilizaram a SDA aplicada para a economia brasileira, destacam-se: Sesso Filho et al. (2010), Araújo Júnior e Tavares (2011), Silva e Perobelli (2012), Cabral e Perobelli (2012) e Moreira e Ribeiro (2013). A maioria desses estudos, no entanto, foi aplicada para modelos nacionais de insumo-produto e utilizou o método de decomposição temporal. Desse modo, no intuito de buscar novos resultados, diversificar e aprofundar tal metodologia, o presente artigo aplica um método de decomposição estrutural espacial para um modelo inter-regional.

O uso dessa metodologia do ponto de vista espacial torna-se interessante quando aplicada em economias que apresentam fortes padrões de heterogeneidade, como é o caso da brasileira, ou, mais especificamente, para uma determinada macrorregião. A desigualdade intrarregional do Nordeste, por exemplo, segundo Sá (2009, p. 157), "[...] produz diferenças de oportunidades econômicas, de ocupação e de condições de vida para a imensa maioria da população, tão danosas quanto as diferenças inter-regionais brasileiras.".

O objetivo deste artigo é investigar as disparidades intrarregionais da região Nordeste, a partir da identificação de semelhanças e diferenças na estrutura produtiva entre seus nove estados. Para isso, aplica-se a análise de decomposição espacial inspirada em Feldman et al. (1987) e adaptada de acordo com as propostas de Dietzenbacher et al. (2000) e Jackson e Dzikowski (2002). A base de dados é

$1 \quad$ Para uma visão geral dessa temática na literatura, ver Feldman et al. (1987), Dietzenbacher e Los (1998) e Miller e Blair (2009). 
a MIP construída para o ano de 2004, com 111 setores econômicos e 10 regiões (nove estados do Nordeste e resto do Brasil), estimada por Guilhoto et al. (2010).

A principal contribuição deste artigo, portanto, é apresentar novas evidências sobre as diferenças regionais no Nordeste. Mais especificamente, é feita uma avaliação das diferenças sobre a ótica do consumo (demanda final) e sobre a ótica do consumo intermediário (relações intersetoriais).

A estrutura do presente trabalho, além desta introdução, está dividida em mais quatro seções: a segunda discute a disparidade regional no Brasil e no Nordeste; a terceira descreve a base de dados e a metodologia; a quarta discute os principais resultados; e, por fim, a quinta tece as considerações finais.

\section{Disparidades Regionais no Brasil}

A dimensão geográfica do território brasileiro e seus problemas persistentes de pobreza, distribuição de renda e disparidades regionais motivaram diversos estudos no país. Nas palavras de Furtado (1960, p. 10), “[...] imenso contínuo territorial, dotado de unidade política e cultural, mas descontínuo e heterogêneo do ponto de vista econômico". Na tentativa de avaliar tais problemas, abordagens econométricas foram utilizadas em larga escala, principalmente nos anos 1990, no intuito de identificar processos de convergência de renda no Brasil. Ferreira e Diniz (1995) e Azzoni (2001), por exemplo, apontam convergência entre as rendas per capita dos estados brasileiros entre 1970 e 1995.

Seguindo outra abordagem, Williamson (1965), inspirado no trabalho de $\mathrm{Ku}$ znets (1955), tenta comprovar sua teoria do U-invertido. Para Williamson (1965), as causas das disparidades regionais estavam baseadas na distribuição dos recursos naturais entre regiões, na migração do trabalho, no movimento do capital e nas políticas de governo. Esse coeficiente foi utilizado em diversos estudos aplicados para a economia brasileira, entre os quais podem-se destacar Haddad e Andrade (1974), Redwood III (1977), Souza (1993), Nasser (2000), Azzoni (2001), Cavalcante (2003) e Ribeiro (2015). Apesar da magnitude dos coeficientes diferirem um pouco, talvez por causa da utilização de diferentes fontes de dados, as conclusões dos referidos autores convergem para a hipótese de que, ao longo do tempo, está ocorrendo redução das disparidades regionais no Brasil.

Apesar da desigualdade regional no Brasil ainda ser uma das maiores do mundo (BAER, 2007; SHANKAR; SHAH, 2003), a mesma vem apresentando redução nas últimas décadas, (AZZONI, 2001; DINIZ 1993, 2006; SILVEIRA NETO; AZZONI, 2011, 2012). Para esses autores, são quatro os principais fatores que explicam essa redução: a) desconcentração da produção industrial; b) estabilização monetária em 1994; c) política de valorização do salário mínimo; e d) programas governamentais de transferência de renda. 
A Figura 1 apresenta o índice de Gini para as macrorregiões brasileiras, entre 1990 e 2014. Assim como o Brasil, todas as suas regiões apresentam redução das disparidades nas duas últimas décadas. No entanto, o Nordeste, ainda que por uma sutil diferença, é a macrorregião brasileira mais desigual em termos intrarregionais.

Figura 1 - Coeficiente de Gini para as macrorregiões brasileiras (1990-2014)

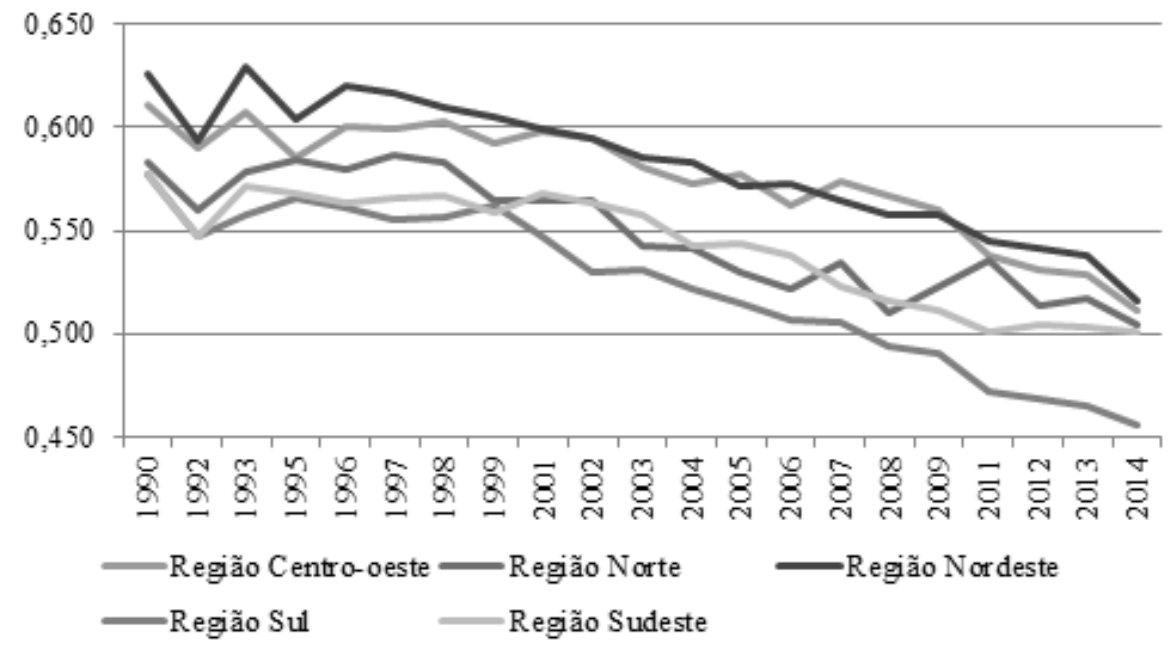

Fonte: Elaboração própria a partir de dados do Ipeadata (1990-2014).

Para Guimarães Neto (1997), essa heterogeneidade presente na região é resultado do próprio desenvolvimento da economia brasileira e da integração produtiva inter-regional. Historicamente, segundo Araújo (2004), nunca existiu um Nordeste homogêneo do ponto de vista econômico, principalmente em decorrência dos diferentes processos de ocupação do território. O que se observa é uma elevada desigualdade em relação às condições sociais da população e muitas tendências gerais que não se reproduzem de forma homogênea entre os estados ou zonas urbanas e rurais da região, gerando concentração da riqueza e forte contraste social. Por outro lado, as regiões Sul e Sudeste são as que apresentaram menores coeficientes e, portanto, são mais homogêneas em termos de diferenças do nível de renda.

Por meio da Tabela 1, é mais fácil perceber um processo de redução das disparidades entre os estados nordestinos. O PIB per capita da Bahia em 1990, estado mais rico da região, era mais do que o dobro do PIB per capita do estado mais pobre, Piauí. Já em 2010, essa diferença cai para aproximadamente 56\%. Não obstante, vale salientar que, de acordo com Diniz (2013, p. 7), “[...] a renda per capita média da região ainda é menos que a metade da média nacional e, pela 
comparação entre os estados mais pobres (Maranhão e Piauí) e o mais rico do país (São Paulo), a diferença é de quase cinco vezes.".

Tabela 1 - PIB per capita: 1990-2010 (em R\$ mil de 2000)

\begin{tabular}{lccccc}
\hline Região e estados do Nordeste & $\mathbf{1 9 9 0}$ & $\mathbf{1 9 9 5}$ & $\mathbf{2 0 0 0}$ & $\mathbf{2 0 0 5}$ & $\mathbf{2 0 1 0}$ \\
\hline Nordeste & $\mathbf{2 . 7 8}$ & $\mathbf{2 . 7 6}$ & $\mathbf{3 . 0 0}$ & $\mathbf{3 . 4 7}$ & $\mathbf{4 . 2 7}$ \\
Alagoas & 2.58 & 2.28 & 2.47 & 2.95 & 3.51 \\
Bahia & 3.47 & 3.24 & 3.67 & 4.15 & 4.91 \\
Ceará & 2.34 & 2.73 & 2.77 & 3.19 & 4.11 \\
Maranhão & 1.49 & 1.44 & 1.62 & 2.62 & 3.07 \\
Paraíba & 2.43 & 2.42 & 2.67 & 2.96 & 3.78 \\
Pernambuco & 3.43 & 3.50 & 3.65 & 3.74 & 4.83 \\
Piauí & 1.59 & 1.77 & 1.86 & 2.33 & 3.16 \\
Rio Grande do Norte & 2.73 & 2.74 & 3.32 & 3.75 & 4.55 \\
Sergipe & 3.53 & 3.25 & 3.28 & 4.30 & 5.16 \\
Norte & $\mathbf{4 . 5 0}$ & $\mathbf{3 . 9 2}$ & $\mathbf{3 . 8 7}$ & $\mathbf{4 . 5 6}$ & $\mathbf{5 . 6 7}$ \\
Centro-Oeste & $\mathbf{5 . 0 1}$ & $\mathbf{5 . 5 1}$ & $\mathbf{6 . 5 0}$ & $\mathbf{9 . 2 0}$ & $\mathbf{1 1 . 1 3}$ \\
Sul & $\mathbf{7 . 5 6}$ & $\mathbf{7 . 3 8}$ & $\mathbf{7 . 6 5}$ & $\mathbf{8 . 3 2}$ & $\mathbf{1 0 . 1 4}$ \\
Sudeste & $\mathbf{8 . 6 1}$ & $\mathbf{8 . 4 6}$ & $\mathbf{8 . 7 1}$ & $\mathbf{9 . 7 5}$ & $\mathbf{1 1 . 6 0}$ \\
\hline
\end{tabular}

Fonte: Elaboração própria a partir de Ipeadata (1990-2010).

Em termos macrorregionais, a estrutura espacial heterogênea se torna mais

presente. É possível ver, na Tabela 1, que o Nordeste tem o menor PIB per capita em todo o período de análise e que a região Centro-Oeste tem o maior crescimento do PIB per capita no período analisado. Mais que isso, o PIB per capita das regiões Centro-Oeste, Sul e Sudeste é mais do que o dobro do apresentado pelo Nordeste.

\section{Base de Dados e Metodologia}

Esta seção tem por objetivo apresentar a base de dados, bem como descrever a metodologia utilizada neste estudo.

\subsection{Base de Dados}

A base de dados é referente à MIP do Nordeste e seus estados, ano base de 2004 e estrutura setorial de 169 produtos e 111 atividades (GUILHOTO et al. 2010). O grau de abertura dessa MIP oferece resultados mais detalhados no que se refere 
principalmente a setores associados à agropecuária, indústria alimentícia, comércio, serviços de utilidade pública e transportes.

Essa base de dados produz um tradeoff entre defasagem temporal e riqueza de detalhamento. Ou seja, embora a MIP utilizada seja referente ao ano de 2004, possui um detalhamento setorial que favorece a análise.

\subsection{Metodologia}

A técnica de decomposição desenvolvida neste trabalho é uma variação da implementada por Feldman et al. (1987). A utilização da análise de decomposição espacial permitirá identificar mudanças na estrutura de produção de cada estado nordestino em relação à média da região.

\subsubsection{Decomposição Temporal nos Modelos de Insumo-Produto}

A equação 1 representa a solução do modelo de insumo-produto proposto por Leontief:

$$
X=(I-A)^{-1} f
$$

em que $X$ é o vetor de produção setorial total, $A$ é a matriz de coeficientes técnicos e f é o vetor de demanda final. Sendo $B$ a matriz inversa de Leontief, a equação 1 pode ser reescrita como:

$$
X=B \cdot f
$$

A equação 2 pode ser escrita de forma a evidenciar a questão temporal, isto é, indicando com o subscrito zero o período inicial e com o subscrito um o período final da análise:

$$
\begin{aligned}
& X_{0}=B_{0} \cdot f_{0} \\
& X_{1}=B_{1} \cdot f_{1}
\end{aligned}
$$

As mudanças na produção podem ser decompostas da seguinte forma: subtraindo-se a equação 3 da equação 4 e depois adicionando-se e subtraindo-se o termo $C_{1} f_{0}$ no lado direito da equação e rearranjando-se os termos:

$$
X_{1}-X_{0}=C_{1}\left(f_{1}-f_{0}\right)+\left(C_{1}-C_{0}\right) f_{0}
$$




\subsubsection{Decomposição Espacial da Produção}

A derivação da análise de decomposição espacial utilizada é baseada no trabalho de Jackson e Dzikowski (2002) e segue a formulação temporal apresentada na subseção anterior. Para se implementar a decomposição espacial, adicionam-se subscritos na equação 2 , que representam cada estado (s) e a média da economia $(m)$ :

$$
\begin{aligned}
& X_{s}=C_{s} \cdot f_{s} \\
& X_{m}=C_{m} \cdot f_{m}
\end{aligned}
$$

É oportuno definir o conceito de "média da economia". Para o presente estudo, foi calculado um vetor de demanda final $\left(f_{m}\right)$ a partir da média das demandas finais dos nove estados do Nordeste, bem como realizado o mesmo procedimento (para cada coeficiente técnico) para se calcular uma matriz inversa de Leontief "média" do Nordeste $\left(C_{m}\right)$.

De maneira análoga à decomposição temporal, subtrai-se a equação 6 da equação 7 e depois adiciona-se e subtrai-se o termo $C_{m} f_{s}$ no lado direito da equação:

$$
X_{m}-X_{s}=C_{m} f_{m}-C_{s} f_{s}+C_{m} f_{s}-C_{m} f_{s}
$$

ou

$$
X_{m}-X_{s}=C_{m}\left(f_{m}-f_{s}\right)+\left(C_{m}-C_{s}\right) f_{s}
$$

O primeiro termo do lado direito da equação 9 indica a diferença na produção devida às diferenças na distribuição regional da demanda final ponderada pela média da estrutura intersetorial, . O segundo termo permite calcular a parcela da diferença no produto, que é devida às diferenças nos coeficientes interindustriais, ponderado pela estrutura de demanda final do estado, $\mathrm{f}_{\mathrm{s}}$.

A análise de decomposição espacial pode ser apresentada de outra maneira, como pode ser visto por meio das equações 10 e 11 :

$$
X_{m}-X_{s}=C_{m} f_{m}-C_{s} f_{m}+C_{s} f_{m}-C_{s} f_{s}
$$

ou

$$
X_{m}-X_{s}=C_{s}\left(f_{m}-f_{s}\right)+\left(C_{m}-C_{s}\right) f_{m}
$$


O primeiro termo do lado direito da equação 11 mostra a diferença na produção setorial em duas regiões como resultante das diferenças na demanda final, ponderadas pela distribuição interindustrial de cada estado, . O segundo termo mede a parcela da produção industrial, que é devida à diferença nos coeficientes interindustriais ponderados pela demanda final.

Miller e Blair (2009) mostram que a análise de decomposição pode ser desenvolvida de diversas maneiras. No entanto, adota-se o método proposto por Dietzenbacher et al. (2000), o qual utiliza uma combinação a partir das equações 9 e 11. Essa combinação mede, para cada indústria $i$, a diferença na produção setorial total como resultante das diferenças na demanda final:

$$
\frac{1}{2}\left[\sum_{j=1}^{n} C_{i j}{ }^{M}\left(f_{j}^{s}-f_{j}^{M}\right)+\sum_{j=1}^{n} C_{i j}{ }^{S}\left(f_{j}^{S}-f_{j}^{M}\right)\right]
$$

em que $C_{i j}^{M}$ e $C_{i j}^{S}$ são elementos da matriz inversa de Leontief para a região tomada como média e para o estado sob investigação, respectivamente. $O$ vetor resultante da equação 12 indica uma medida de qual a contribuição das diferenças na demanda final para as diferenças na produção setorial entre a região tomada como média e a região em análise. Para se flexibilizar a hipótese de escolha da "média" da região, substitui-se o $C_{i j}{ }^{M}$ por $C_{i j}{ }^{1}, \ldots, C_{i j}{ }^{9}$, os quais representam a matriz inversa de Leontief de cada um dos nove estados da região Nordeste, e $C_{i j}{ }^{S}$ representa a inversa de Leontief total. Assim, a equação 12 pode ser reescrita como:

$$
\frac{1}{2}\left[\sum_{j=1}^{n} C_{i j}^{1}\left(f_{j}^{s}-f_{j}^{1}\right)-\ldots-\sum_{j=1}^{n} C_{i j}{ }^{9}\left(f_{j}^{s}-f_{j}^{9}\right)+\sum_{j=1}^{n} C_{i j}{ }^{s}\left(f_{j}^{s}-f_{j}^{1}\right)-\ldots-\sum_{j=1}^{n} C_{i j}^{s}\left(f_{j}^{s}-f_{j}^{9}\right)\right]
$$

É possível, ainda, utilizarem-se as equações 9 e 11 para se obter, para cada indústria $i$, uma medida que representa a diferença na produção setorial total como resultante das diferenças nos coeficientes intersetoriais:

$$
\frac{1}{2}\left[\sum_{j=1}^{n}\left(C_{i j}{ }^{S}-C_{i j}{ }^{M}\right) f_{j}^{s}+\sum_{j=1}^{n}\left(C_{i j}{ }^{S}-C_{i j}{ }^{M}\right) f_{j}{ }^{M}\right]
$$

O resultado da equação 14 permite ao pesquisador calcular qual é a contribuição das diferenças na estrutura interindustrial para as diferenças na produção setorial entre a região média e o estado em análise. 


\subsubsection{Campo de Influência}

O método do campo de influência foi desenvolvido por Sonis e Hewings (1991). A partir dessa técnica, é possível visualizar os setores com maiores elos de encadeamento em uma determina estrutura produtiva. Em outras palavras, as compras e vendas dos setores com maior campo influenciam as relações intersetoriais das demais atividades.

Para o seu cálculo, utiliza-se a matriz de coeficientes técnicos $(A)$, uma matriz de variações incrementais nos coeficientes $(E)$ e a matriz inversa de Leontief $(B)$. Parte-se de uma pequena variação ${ }^{2} \mathcal{E}$, em cada $a_{i j}$ isoladamente, ou seja, $\Delta A$ é uma matriz $E=\left|\varepsilon_{i j}\right|$, tal que:

$$
\varepsilon_{i j}=\left\{\begin{array}{l}
\varepsilon \text { se } i=i_{1} \text { e } j=j_{1} \\
0 \text { se } i \neq i_{1} \text { e } j \neq j_{1}
\end{array}\right.
$$

A matriz inversa de Leontief é recalculada considerando-se a variação $\varepsilon$. Logo, o campo de influência de cada coeficiente é retratado na equação 16 , ao passo que a influência total de cada coeficiente técnico da MIP é calculada por meio da equação 17:

$$
\begin{gathered}
F\left(\varepsilon_{i j}\right)=\frac{B^{*}-B}{\varepsilon_{i j}} \\
S_{i j}=\sum_{k=1}^{n} \sum_{l=1}^{n}\left[f_{k l}\left(\varepsilon_{i j}\right)\right]^{2}
\end{gathered}
$$

Quanto maior for $S_{\ddot{y}}$, portanto maior será o campo de influência do coeficiente $a_{i j}$ sobre a estrutura produtiva.

\section{Resultados e Discussões}

O uso da análise de decomposição espacial identificará as diferenças e/ou similaridades intrarregionais entre os estados nordestinos, bem como dos seus setores econômicos. Os resultados apresentados nesta seção são derivados das equações 12, 13 e 14. As duas primeiras revelam se a demanda final desempenha, ou não, papel relevante na diferenciação da produção de cada setor em cada estado.

$\overline{2 \quad \text { Adotou-se um } e}=0,001$. 
Por outro lado, os resultados obtidos a partir da equação 14 indicarão quais setores são mais fortemente orientados às relações intersetoriais quando comparados à média regional.

Os resultados iniciais são para nove unidades espaciais e 111 setores produtivos. A fim de se dar maior ênfase aos resultados setoriais, a seguinte estratégia foi adotada: utilizaram-se os resultados do campo de influência e, para cada estado, identificaram-se os 31 setores (ver Tabela 2) que apresentam maior efeito de encadeamento na estrutura produtiva dos estados nordestinos. Não obstante, por meio da SDA, será possível avançar na análise sistêmica da estrutura de produção e diferenças regionais no Nordeste.

Tabela 2 - Setores econômicos com maior encadeamento nos estados do Nordeste

\begin{tabular}{|c|c|c|c|}
\hline 1 & Cana-de-açúcar & 17 & Adubos e fertilizantes \\
\hline 2 & Bovinos & 18 & Fabricação de aço e derivados \\
\hline 3 & Aves & 19 & $\begin{array}{l}\text { Máquinas para escritório e equipa- } \\
\text { mentos de informática }\end{array}$ \\
\hline 4 & Abate de bovinos & 20 & $\begin{array}{l}\text { Material eletrônico e equipamentos } \\
\text { de comunicações }\end{array}$ \\
\hline 5 & Abate de aves & 21 & $\begin{array}{l}\text { Peças e acessórios para veículos } \\
\text { automotores }\end{array}$ \\
\hline 6 & Fabricação de óleos vegetais & 22 & Outros equipamentos de transporte \\
\hline 7 & Indústria de laticínios & 23 & Comércio atacadista \\
\hline 8 & Fabricação de açúcar & 24 & Transporte rodoviário carga \\
\hline 9 & Indústria do café & 25 & Transporte aéreo carga \\
\hline 10 & Produtos do fumo & 26 & Transporte dutoviário carga \\
\hline 11 & Têxteis & 27 & Serviços de telefonia móvel \\
\hline 12 & Artefatos de couro e calçados & 28 & Serviços de telefonia fixa \\
\hline 13 & $\begin{array}{l}\text { Produtos de madeira (exclusive } \\
\text { móveis) }\end{array}$ & 29 & Outros serviços de informação \\
\hline 14 & $\begin{array}{l}\text { Fabricação de celulose e pasta } \\
\text { mecânica }\end{array}$ & 30 & Intermediação financeira e seguros \\
\hline 15 & $\begin{array}{l}\text { Fabricação de papel, papelão e } \\
\text { artefatos de papel }\end{array}$ & 31 & Serviços prestados às empresas \\
\hline 16 & Refino de petróleo e coque & & \\
\hline
\end{tabular}

Fonte: Elaboração própria.

A Tabela 2 revela os setores mais encadeados entre os estados nordestinos. Do um ao três aparecem atividades pertencentes à agropecuária, do quatro ao 22 
têm-se setores vinculados à indústria de transformação e do 23 ao 31 aparecem atividades ligadas ao segmento de serviços. A partir dessas atividades, a Figura 2 revela o resultado da decomposição espacial utilizando a região Nordeste como média (ver equação 13) e a Figura 3 sem a utilização do conceito de média, ou seja, obtido por meio da equação 14 .

Figura 2 - Diferenças no produto setorial a partir da demanda final: média regional

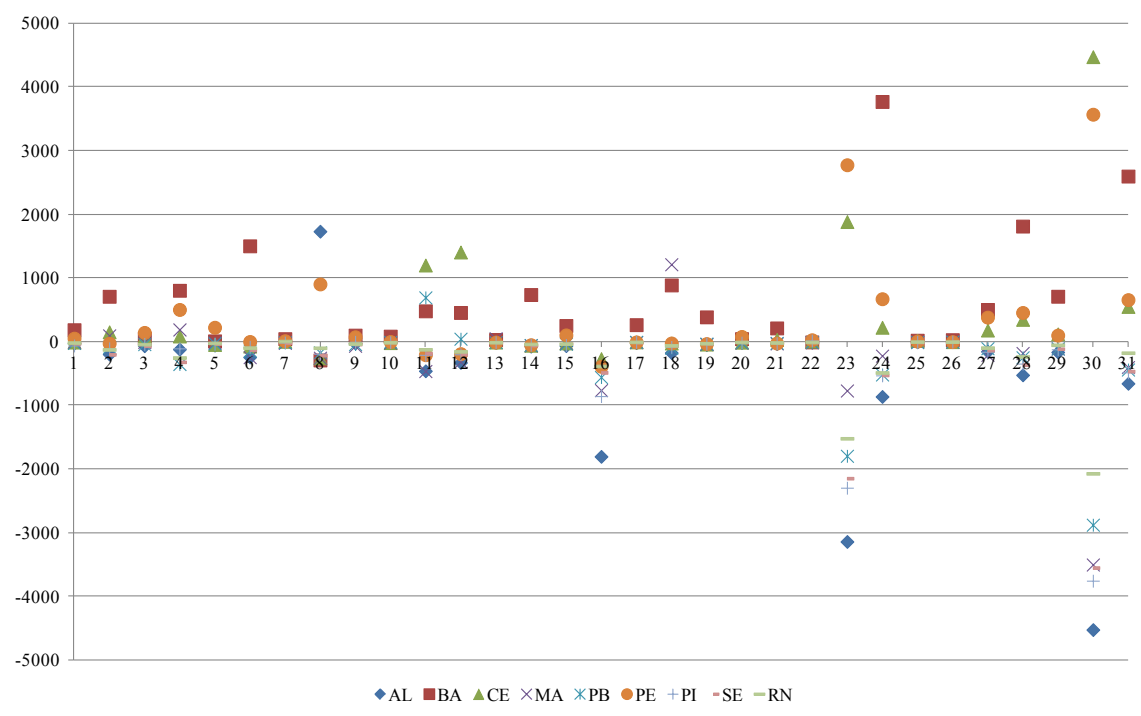

Fonte: Elaboração própria a partir da base na matriz de insumo-produto (2004).

Nota: Os setores 16, 23 e 30 da Bahia apresentaram resultados muito superiores aos dos demais (outliers) e, por esse motivo, não aparecem no gráfico. 
Figura 3 - Diferenças no produto setorial a partir da demanda final

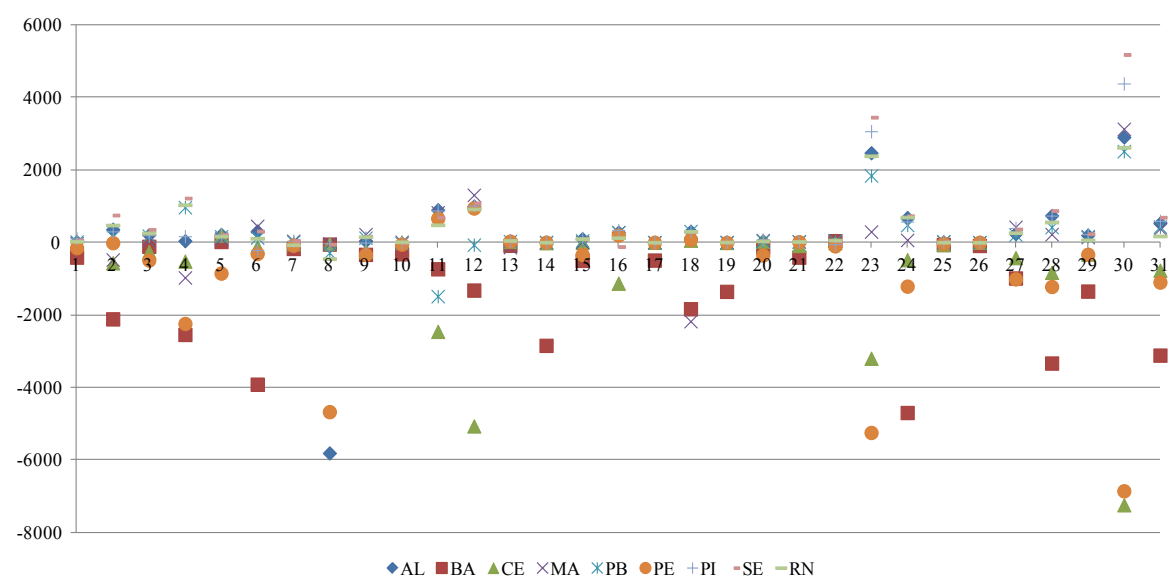

Fonte: Elaboração própria a partir da base na matriz de insumo-produto (2004).

Nota: Os setores 16, 23 e 30 da Bahia apresentaram resultados muito superiores aos dos demais (outliers) e, por esse motivo, não aparecem no gráfico.

As Figuras 2 e 3 mostram, em seu eixo horizontal, os 31 setores produtivos com maior encadeamento da MIP e, no seu eixo vertical, as diferenças (em $R \$$ milhões de 2004) na estrutura produtiva devido à variação na demanda final. Percebe-se que os resultados nas duas figuras basicamente são os mesmos. A diferença está na visualização: enquanto no primeiro os setores que têm variações na produção explicadas pela demanda final ficam acima do eixo (valores positivos), no segundo ficam abaixo (valores negativos). Assim, é possível afirmar que, nos estados da Bahia, Pernambuco e Ceará, a demanda final desempenha papel relevante na diferenciação do produto, para a maioria das atividades. Esse resultado é em parte explicado pela participação desses estados no PIB da região Nordeste. Esse resultado fica mais evidente para os setores 23 a 31, ou seja, para o segmento de serviços. No outro extremo, estão os resultados para os estados de Alagoas, Maranhão, Paraíba, Piauí, Sergipe e Rio Grande do Norte, principalmente em relação aos setores 16, 23, 24 e 30.

À exceção dos estados da Bahia, Ceará e Pernambuco, percebe-se que a demanda final não desempenha papel relevante na diferenciação do produto para os setores 1 a 10 dos demais estados, os quais abrangem atividades da agropecuária, da indústria de alimentos e da produção de fumo.

Para entender melhor esses resultados, a Figura 4 apresenta a estrutura de participação dos componentes da demanda final para cada estado no Nordeste em 2004. Percebe-se que o consumo das famílias (C) responde pela maior participação em todos os estados nordestinos, em média 55\%. No sentido oposto, as exportações representam as menores participações (7\%, em média), ou seja, o 
componente doméstico responde por $93 \%$ em média da demanda total. De acordo com Medeiros (2015, p. 52), "[...] a evolução dos padrões de consumo constitui, ao lado do progresso técnico, um vetor fundamental do processo de mudança estrutural, que caracteriza o desenvolvimento econômico.". Embora na discussão de crescimento as medidas de produto per capita sejam muito utilizadas, ${ }^{3}$ isso não se reflete em uma boa medida de consumo dos indivíduos/famílias ou de desenvolvimento econômico (BRESSER-PEREIRA, 2004). O aumento dos salários e do padrão de consumo regionais, por outro lado, pode ser um indicativo de maior desenvolvimento econômico. Portanto, o consumo das famílias pode ser considerado proxy para crescimento econômico e para aquecimento da economia regional.

No sentido de reforçar tal afirmação, resultados econométricos estimados por Oreiro et al. (2010) mostram que, entre 1991 e 2005, 95\% do aumento do PIB real da economia brasileira foi explicado por variáveis da demanda agregada. De forma categórica esses autores afirmam que os mecanismos que restringem o crescimento econômico de longo prazo devem ser investigados pelo lado da demanda, e não pelo da oferta da economia.

Figura 4 - Participação dos componentes da demanda final nos estados do Nordeste

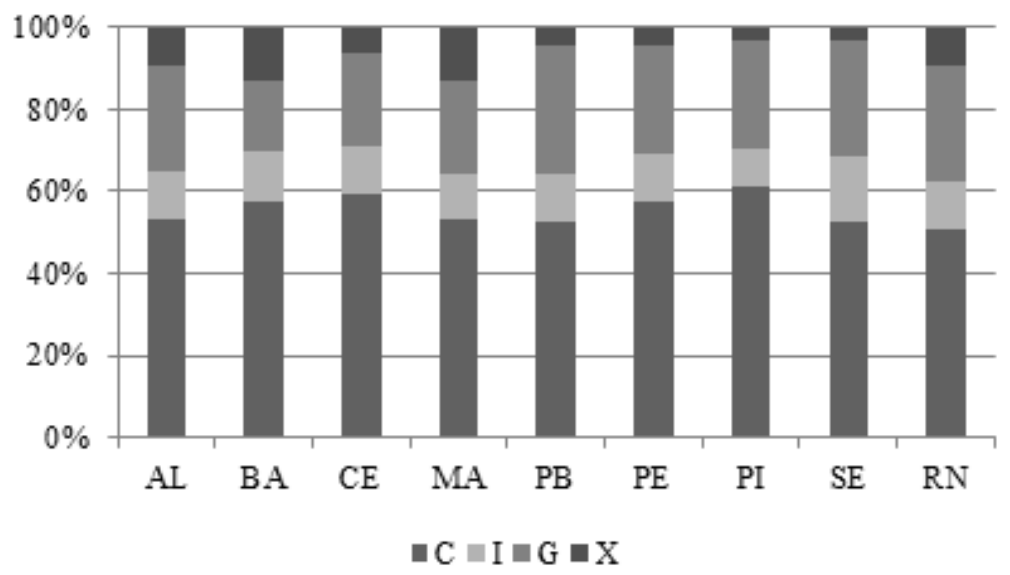

Fonte: Elaboração própria a partir da base na matriz de insumo-produto (2004).

Em relação aos resultados mais específicos das Figuras 2 e 3, destaca-se o setor 8 , indústria do açúcar de Alagoas e Pernambuco, que também apresentou resultado muito inferior à média, bem como foram os únicos estados que obtiveram tal resultado para esse setor. Vale salientar que esses dois estados são os maiores produtores de açúcar da região. Para a produção de açúcar alagoana, $46 \%$ da sua demanda final é exportada, 49,5\% é demandada domesticamente pelas famílias e

3 Ver Solow (1956) e Barro (1991). 
4,4\% é utilizada para formação bruta de capital fixo. Para Pernambuco, a estrutura de demanda final já é bem diferente: somente 12\% é exportado, 80,9\% é consumido internamente e $7 \%$ é destinado para formação de capital fixo. ${ }^{4}$

A indústria têxtil (11) dos estados da Bahia, Ceará e Paraíba também se destacou dos demais, isto é, para esses estados a demanda final desempenha papel de diferenciação da produção têxtil. Para esse segmento, a estrutura de demanda final é similar para os estados da Bahia e Paraíba: em média 58,7\% é destinado para exportação e 41,3\% para o consumo das famílias. Já para o Ceará, essa estrutura se inverte, ou seja, $51,9 \%$ para o consumo das famílias e $48,1 \%$ para a exportação.

Destaca-se o setor 16, refino de petróleo e coque da Bahia: para esse segmento é possível evidenciar o papel desempenhado pela demanda final. Importante salientar que, para esse setor, somente a demanda final da Bahia desempenha papel relevante na diferenciação do produto setorial. Esse resultado pode ser explicado, em parte, pela presença da Refinaria Landulpho Alves (RLAM) e do Polo Petroquímico de Camaçari, fazendo da Bahia um importante fornecedor regional de produtos derivados do refino de petróleo. Para esse setor, $63,4 \%$ da sua demanda final é destinada para exportação e 36,6\% é consumida internamente pelas famílias.

A Bahia e o Maranhão são os únicos estados a apresentarem importante papel da demanda final na diferenciação da produção do setor 18, fabricação de aço e derivados. Salienta-se que o Maranhão tem um importante complexo mínero-metalúrgico que, segundo Carvalho (2008), contribui para a dinâmica regional. Vale salientar que para ambos os estados essa atividade se destina quase que completamente para abastecer o mercado externo com participações da exportação na demanda final de $95,5 \%$ e $99,9 \%$, respectivamente.

Em suma, os estados da Bahia, Ceará e Pernambuco, como dito anteriormente, apresentaram melhores resultados. Esses estados que são maiores e mais ricos se destacam dos demais devido à assimetria entre as economias dessa região. Tais unidades da federação detêm melhores condições financeiras, o que permitiu que realizassem investimentos em infraestrutura, o desenvolvimento de polos agrícolas e industriais, o recebimento de importantes empreendimentos imobiliários para exploração turística, etc.

Ficou evidenciado que a demanda final dos setores associados ao segmento de serviços, em geral, desempenha papel relevante na diferenciação do produto. Isso pode estar relacionado ao fato dos serviços terem aumentado sua participação no valor adicionado bruto do Nordeste, como mostra a Figura 5.

4 Para as análises de destino por componente da demanda final, foi desconsiderado o item variação de estoques. 
Figura 5 - Participação setorial no valor adicionado bruto do Nordeste

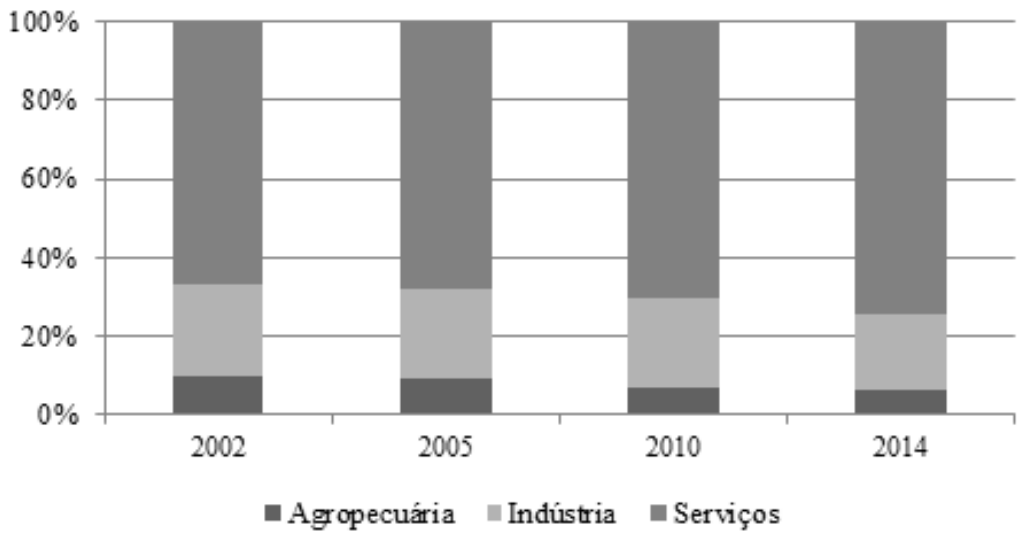

Fonte: Elaboração própria a partir de dados das Contas Regionais do IBGE (IBGE, 2016).

A partir dos resultados, pode-se fazer uma analogia com a existência de "vários" Nordestes, como apontado por Araújo (2004), embora em escala estadual. Percebe-se que os setores baianos, cearenses e pernambucanos são, na sua maioria, mais dinâmicos, devido em parte à concentração de investimentos nessas áreas (LIMA, 1994; LIMA; SIMÕES, 2010), ao passo que os demais estados apresentam, majoritariamente, setores pouco dinâmicos ou mesmo estagnados. Caso as forças de mercado sejam o único fator determinante nas alocações dos empreendimentos, é de se esperar, segundo Guimarães Neto (1997), que as regióes mais industrializadas venham a concentrá-los. Para esse autor, isso decorre de duas razões: a) maior capacidade tecnológica oriunda da sua complexa estrutura produtiva ou da sua maior integração com o mercado internacional; e b) proximidade de centros de pesquisa.

A interpretação da Figura 6 se restringe à análise das relações intersetoriais dos estados nordestinos comparados com a média da região (equação 14). O procedimento de interpretação é análogo ao que foi feito anteriormente para a demanda final. Basicamente, os setores dos estados que apresentarem valores positivos muito superiores são mais fortemente orientados às relações intersetoriais do que a região tomada como média. Pode-se fazer uma analogia desse resultado com os índices de ligação de Hirschman-Rasmussen, isto é, setores que apresentam fortes relações de interdependência são de extrema importância para as economias regionais, pois impactam um maior número de atividades quando estimulados. 
Figura 6 - Diferenças no produto setorial a partir da estrutura intersetorial

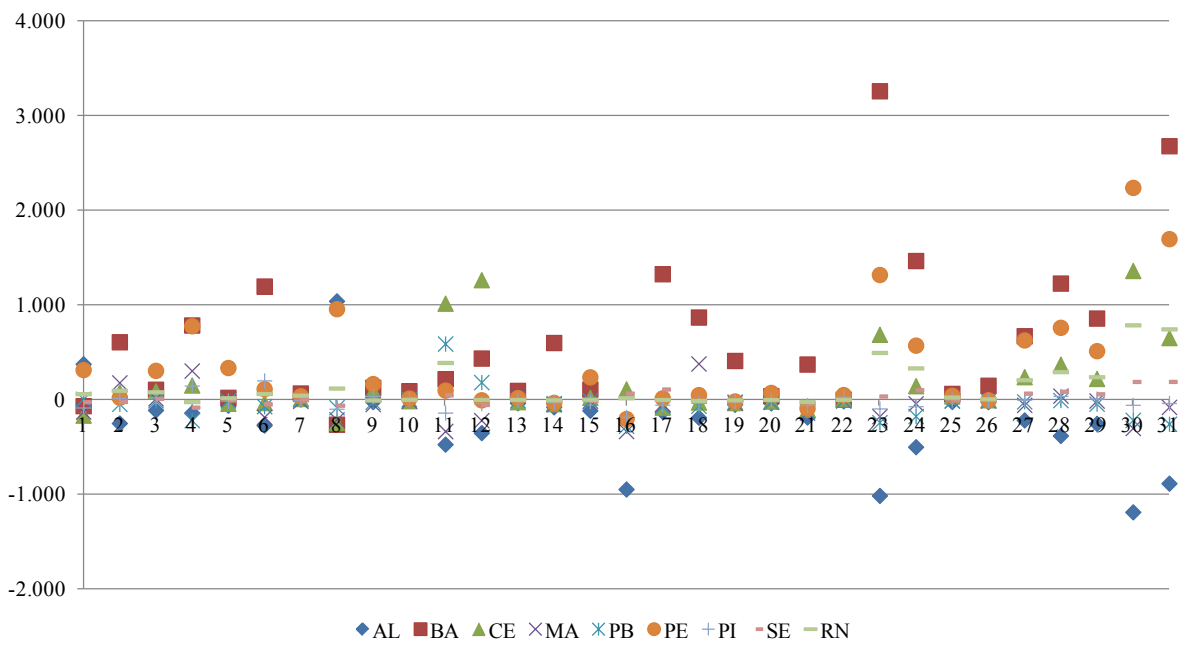

Fonte: Elaboração própria com base na matriz de insumo-produto (2004).

Nota: Os setores 16 e 30 da Bahia apresentaram resultados muito superiores aos dos demais (outliers) e, por esse motivo, não aparecem no gráfico.

Ao se observar a Figura 6 é possível afirmar que os estados da Bahia, Pernambuco e Ceará são aqueles que apresentam maior número de setores-chave nos três segmentos produtivos: agropecuária, indústria e serviços. É interessante destacar que, para esses três estados, os mesmos setores tiveram os maiores valores absolutos: 23, comércio atacadista, 30, intermediação financeira e seguros, e 31 , serviços prestados às empresas.

Os setores baianos 16, refino de petróleo e coque, e 30, intermediação financeira e seguros, apresentaram resultados elevados e, por isso, foram considerados como outliers na Figura 4. A maioria dos resultados positivos para o estado de Sergipe está bem próxima à média. Dentre os 31 setores com maior encadeamento produtivo no Nordeste, a economia sergipana apresentou 14 setores-chave, com destaque para as atividades 30, intermediação financeira e seguros, e 31, serviços prestados às empresas. O estado do Rio Grande do Norte apresentou 21 setores-chave, com destaque também para os setores 30, intermediação financeira e seguros, e 31, serviços prestados às empresas.

O estado do Piauí apresentou apenas nove setores-chave, com destaque para o setor 6, fabricação de óleos vegetais. Alagoas foi o estado nordestino que apresentou menos setores-chave, quatro, com destaque para o 8 , fabricação de açúcar.

Vale destacar que, para os estados da Bahia, Ceará, Pernambuco, Sergipe e Rio Grande do Norte, os setores 30, intermediação financeira e seguros, e 31 , 
serviços prestados às empresas, foram os que apresentam um dos melhores resultados. Por outro lado, esses mesmos setores, nos estados de Alagoas, Maranhão, Paraíba e Piauí, obtiveram os piores resultados.

No intuito de melhor observar o desempenho desses setores em cada um dos estados nordestinos, selecionou-se $10 \%^{5}$ das atividades que apresentaram maior resultado da decomposição da produção. Dos 28 setores, 13 estão localizados na Bahia, sete em Pernambuco, cinco no Ceará, dois no Rio Grande do Norte e um em Alagoas. Os demais estados do Nordeste (Maranhão, Paraíba, Piauí e Sergipe), de acordo com esse critério, não obtiveram nenhum setor. A Tabela 3 indica quais são esses setores, bem como em quais estados estão localizados.

Tabela 3 - Ranking dos setores com maior resultado da decomposição da produção

\begin{tabular}{|c|c|c|c|c|c|c|}
\hline & Setor & $\mathbf{A L}$ & BA & $\mathbf{C E}$ & $\mathbf{P E}$ & $\mathbf{R N}$ \\
\hline 2 & Bovinos & & $\mathrm{X}$ & & & \\
\hline 4 & Abate de bovinos & & $\mathrm{X}$ & & $\mathrm{X}$ & \\
\hline 6 & Fabricação de óleos vegetais & & $\mathrm{X}$ & & & \\
\hline 8 & Fabricação de açúcar & $\mathrm{X}$ & & & $\mathrm{X}$ & \\
\hline 11 & Têxteis & & & $\mathrm{X}$ & & \\
\hline 12 & Artefatos de couro e calçados & & & $\mathrm{X}$ & & \\
\hline 16 & Refino de petróleo e coque & & $\mathrm{X}$ & & & \\
\hline 17 & Adubos e fertilizantes & & $\mathrm{X}$ & & & \\
\hline 18 & Fabricação de aço e derivados & & $\mathrm{X}$ & & & \\
\hline 23 & Comércio atacadista & & $\mathrm{X}$ & $\mathrm{X}$ & $X$ & \\
\hline 24 & Transporte rodoviário carga & & $\mathrm{X}$ & & & \\
\hline 27 & Serviços de telefonia móvel & & $\mathrm{X}$ & & $\mathrm{X}$ & \\
\hline 28 & Serviços de telefonia fixa & & $\mathrm{X}$ & & $\mathrm{X}$ & \\
\hline 29 & Outros serviços de informação & & $\mathrm{X}$ & & & \\
\hline 30 & Intermediação financeira e seguros & & $\mathrm{X}$ & $\mathrm{X}$ & $\mathrm{X}$ & $\mathrm{X}$ \\
\hline 31 & Serviços prestados às empresas & & $\mathrm{X}$ & $\mathrm{X}$ & $\mathrm{X}$ & $\mathrm{X}$ \\
\hline
\end{tabular}

Fonte: Elaboração própria a partir da base na matriz de insumo-produto (2004).

De forma resumida, os resultados indicam a formação de dois grupos de estados. O primeiro é formado pelos estados que apresentam as maiores e mais dinâmicas economias, isto é, Bahia, Ceará e Pernambuco. Para a maioria dos setores desses estados, sobretudo no segmento de serviços, a demanda final desempenha

5 Tem-se 31 setores com maior nível de encadeamento produtivo localizados em nove estados diferentes, totalizando 279 atividades. 
papel relevante na diferenciação da atividade produtiva. Além disso, esses três estados apresentam o maior número de setores-chaves na região, os quais têm a capacidade de produzir maiores efeitos multiplicadores devido às suas relações de encadeamento.

O outro grupo é constituído pelos demais estados nordestinos, ou seja, Alagoas, Maranhão, Paraíba, Piauí, Rio Grande do Norte e Sergipe. Nesse caso, observou-se que a demanda final, com algumas exceções, não diferencia a produção das atividades econômicas. Esses estados têm em comum economias mais modestas e menos dinâmicas. Ao contrário do primeiro grupo, esse conjunto de estados apresentou um pequeno número de setores-chave nas suas estruturas produtivas, o que, de certo modo, pode comprometer a integração econômica intraestadual.

\section{Considerações Finais}

Este trabalho, por meio da análise de decomposição espacial, avaliou as semelhanças e disparidades na estrutura produtiva entre os estados da região Nordeste do Brasil. Esse método permitiu identificar mudanças na estrutura de produção de cada estado nordestino em relação à média da região.

Os principais resultados encontrados revelam que a demanda final exerce papel relevante na diferenciação da produção de diversos setores da Bahia, Pernambuco e Ceará, como já era esperado, uma vez que esses estados apresentam as maiores participações no PIB regional e são o principal destino regional das vendas e principal origem regional das compras dos setores produtivos. Esse resultado é observado principalmente no segmento de serviços. Por outro lado, a demanda final não diferencia o produto de algumas atividades da agropecuária, de alguns setores da indústria alimentícia e da produção de fumo, uma vez que se observou padrão similar entre os estados do Nordeste. Para os estados de Alagoas, Sergipe, Piauí e Paraíba, principalmente, a demanda final não explica diferenças nos seus níveis de produto.

Este trabalho ainda identificou um conjunto de setores que apresentam fortes encadeamentos produtivos na região, os quais podem ser alvos de políticas, uma vez que investimentos alocados nessas atividades podem gerar maiores efeitos multiplicadores para a economia como um todo.

Esses resultados mostram a importância de se fomentar políticas de integração produtiva em escalas intra e interestadual na região Nordeste. Em outras palavras, deve-se estimular o fortalecimento das relações de comércio entre os setores nordestinos na medida em que se gerem spillovers intrarregionais quando, por exemplo, alguma cidade ou estado localizados na região sofrer qualquer tipo de intervenção como, por exemplo, instalação de novas unidades industriais. 
Associado a isso, dada a importância da demanda final como driver para o crescimento econômico, é preciso estimular políticas de fomento ao mercado interno regional. Ou seja, incentivar setores fornecedores de insumos para segmentos estratégicos do Nordeste a se instalarem na própria região e, desse modo, aproximar-se do paradigma da "causação circular cumulativa" de Myrdal.

É importante destacar que a Política Nacional de Desenvolvimento Regional (PNDR), instaurada pelo Decreto $\mathrm{n}^{\circ}$ 6.047, de 22 de fevereiro de 2007 (BRASIL, 2007), não tem como objetivos explícitos os pontos levantados anteriormente. Essa política tem como meta a redução das desigualdades regionais no Brasil. Dessa forma, é imprescindível novos eixos de atuação da política em curso ou criação de novas políticas.

Como agenda de pesquisa, é importante avaliar eventuais mudanças estruturais na região por meio da análise dos coeficientes técnicos. Para tanto, precisa-se de pelo menos duas MIP. A partir disso, pode-se encontrar novos resultados e novos desdobramentos de políticas.

\section{Referências}

ARAÚJO JÚNIOR, I. T.; TAVARES, F. B. Mudanças estruturais no Nordeste entre 1997 e 2004: uma análise de insumo-produto. Revista Econômica do Nordeste, Fortaleza, v. 42, n. 4, p. 697-711, 2011.

ARAÚJO, T. B. Nordeste: heranças, oportunidades e desafios. Revista Teoria e Debate, São Paulo, n. 77, p. 1-4, 2008. 2004 . . Northest, Northests: what Northest. Latin American Perspectives, v. 31, n. 2, p. 16-41,

AZZONI, C. R. Concentração regional e dispersão das rendas per capita estaduais: análise a partir de séries históricas estaduais de PIB, 1939-1995. Estudos Econômicos, São Paulo, v. 27, n. 3, p.341-393, 1997.

. Economic growth and regional income inequality in Brazil. The Annals of Regional Science, v. 35, n. 1, p. 133-152, 2001.

BAER, W. Brazilian economy: growth and development. 6. ed. Boulder, CO: Lynne Rienner, 2007.

BARRO, R. J. Economic growth in a cross section of countries. Quarterly Journal of Economics, v. 106, n. 2, p. 407-443, 1991.

BLAIR, P.; WYCKOFF, A. The changing structure of the U.S. economy: an input-output analysis. In: MILLER, R.; POLENSKE, K.; ROSE, A. (Eds.). Frontiers of input-output analysis. New York; London: Oxford University Press, 1989. p. 293-307.

BRASIL. Decreto n ${ }^{\circ}$ 6.047. Disponível em < http:/www.planalto.gov.br/ccivil_03/_Ato20072010/2007/Decreto/D6047.htm>. Acesso em: 10 de novembro de 2017, 2007. 
BRESSER-PEREIRA, L. C. O conceito de desenvolvimento do ISEB rediscutido. Dados, Rio de Janeiro, v. 47, n. 1, p. 49-84, 2004.

CABRAL, J. A.; PEROBELLI, F. S. Análise de decomposição estrutural para o setor de saúde brasileiro - 2000-2005. Pesquisa e Planejamento Econômico, Rio de Janeiro, v. 42, n. 3, p. 363402, 2012.

CARTER, A. Structural change in the American economy. Cambridge: Harvard University Press, 1970.

CARVALHO, C. P. O. Nordeste: sinais de um novo padrão de crescimento (2000/2008). Revista Economia Política do Desenvolvimento, Maceió, v. 1, n. 2, p. 7-40, 2008.

CAVALCANTE, L. R. M. T. Desigualdades regionais no Brasil: uma análise do período 19851999. Revista Econômica do Nordeste, Fortaleza, v. 34, n. 3, p. 466-481, 2003.

CHENERY, H.; SHISHIDO, S.; WATANABE, T. The pattern of Japanese growth, 1914-1954. Econometrica, v. 30, n. 1, p. 98-139, 1962.

CHÓLIZ, J. S.; DUARTE, R. The effect of structural change on the self-reliance and interdependence of aggregate sectors: the case of Spain, 1980-1994. Structural Change and Economic Dynamics, v. 17, n. 1, p. 27-45, 2006.

DIETZENBACHER, E.; LOS, B. Structural decomposition techniques: sense and sensitivity. Economic Systems Research, v. 10, n. 4, p. 307-324, 1998.

DINIZ, C. C. A busca de um projeto de nação: o papel do território e das políticas regional e urbana. Revista Economia, Brasília, v. 7, n. 4, p. 1-18, 2006.

. Desenvolvimento poligonal no Brasil: nem desconcentração nem contínua polarização. Nova Economia, Belo Horizonte, v. 3, n. 1, p. 35-64, 1993.

. Dinâmica regional e ordenamento do território brasileiro: desafio e oportunidades. Belo Horizonte: UFMG/Cedeplar, 2013. (Texto para Discussão, n. 471, 2013).

FELDMAN, S.; MCCLAIN, D.; PALMER, K. Sources of structural change in the United States 1963-78: an input-output perspective. Review of Economics and Statistics, v. 69, n. 3, p. 503510, 1987.

FERREIRA, A. H. B.; DINIZ, C. C. Convergência entre as rendas per capita no Brasil. Revista de Economia Política, São Paulo, v. 15, n. 4, p. 38-56, 1995.

FURTADO, C. A. Operação Nordeste. Rio de Janeiro: Instituto Superior de Estudos Brasileiros, 1959.

. Perspectiva da economia brasileira. 2. ed. Rio de Janeiro: MEC/Instituto Superior de

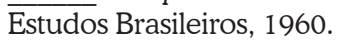

GOMES, G. M.; VERGOLINO, J. R. A macroeconomia do desenvolvimento nordestino: 1960/1994. Brasília: Ipea, 1995. (Texto para Discussão n. 372, 1995).

GUILHOTO, J. J. M. et al. Matriz de insumo-produto do nordeste e estados: metodologia e resultados. Fortaleza: Banco do Nordeste do Brasil, 2010. 
GUIMARÃES NETO, L. Trajetória econômica de uma região periférica. Estudos Avançados, São Paulo, v. 11, n. 29, p. 37-54, 1997.

HADDAD, P. R.; ANDRADE, T. A. Política fiscal e desequilíbrios regionais. Estudos Econômicos, São Paulo, v. 4, n. 1, p. 9-37, 1974.

IBGE. Sistema de Contas Regionais: Brasil 2014. Rio de Janeiro: IBGE, 2016.

IPEADATA. PIB Estadual per capita, 1990-2010. Disponível em: < http://ipeadata.gov.br/ Default.aspx>. Acesso em: 15 dez. 2017.

. Renda - desigualdade - coeficiente de Gini, 1990-2014. Disponível em: < http:// ipeadata.gov.br/Default.aspx>. Acesso em: 05 jan. 2018.

JACKSON, R. W.; DZIKOWSKI, D. A. A spatial decomposition method for assessing regional economic structure. In: HEWINGS, G. J. D; SONIS, M.; BOYCE, D. (Ed). Trade, networks and hierarchies: modelling regional and interregional economies. New York: Springer, 2002. p. 315-327.

KUZNETS, S. Economic growth and income inequality. The American Economic Review, v. 45, n. 1, p. 1-28, 1955.

LEONTIEF, W. Input-output economics. New York: Oxford University Press, 1966.

LIMA, A. C. C.; SIMÕES, R. Centralidade e emprego na região nordeste do Brasil período 1995/2007. Nova Economia, Belo Horizonte, v. 20, n. 1, p. 39-83, 2010.

LIMA, J. P. Economia do Nordeste: tendências das áreas dinâmicas. Revista Análise Econômica, Porto Alegre, v. 12, n. 22, p. 55-73, 1994.

MEDEIROS, C. A. Inserção externa, crescimento e padrões de consumo na economia brasileira. Brasília: Ipea, 2015.

MILLER, R. E.; BLAIR, P. D. Input-output analysis: foundations and extensions. 2 ed. New York: Cambridge University Press, 2009.

MOREIRA, T. M.; RIBEIRO, L. C. S. Mudanças estruturais na economia brasileira entre 20002005 e o novo regime macroeconômico: uma abordagem multissetorial. Revista Economia, Brasília, v. 14, n. 1C, p. 751-780, 2013.

NASSER, B. Economia regional, desigualdade regional no Brasil e o estudo dos eixos nacionais de integração e desenvolvimento. Revista do BNDES, Rio de Janeiro, v. 7, n. 14, p. $145-178,2000$.

OREIRO, J. L.; NAKABASHI, L.; SOUZA, G, J. G. A economia brasileira puxada pela demanda agregada. Revista de Economia Política, São Paulo, v. 20, n. 4, p. 581-603, 2010.

REDWOOD III, J. Evolução recente das disparidades de renda regional no Brasil. Pesquisa e Planejamento Econômico, Rio de Janeiro, v. 7, n. 3, p. 485-550, 1977. 
RIBEIRO, L. C. S. Investimentos estruturantes e desigualdades regionais na região Nordeste. 2015. 204 f. Tese (Doutorado em Ciências Econômicas) - Universidade Federal de Minas Gerais, Belo Horizonte, 2015.

ROSE, A.; CASLER, S. Input-output structural decomposition analysis: a critical appraisal. Economic Systems Research, v. 8, n. 1, p. 33-62, 1996.

SÁ, A. N. M. Diálogos em torno do Nordeste. In: FURTADO, C. et al. O pensamento de Celso Furtado e o Nordeste Hoje. Rio de Janeiro: Contraponto: Centro Internacional Celso Furtado para o Desenvolvimento: Banco do Nordeste do Brasil, 2009. p. 157-178.

SESSO FILHO, U. A. et al. Decomposição estrutural da variação do emprego no Brasil: 1991-2003. Economia Aplicada, Ribeirão Preto, v. 14, n. 1, p. 99-123, 2010.

SHANKAR, R.; SHAH, A. Bridging the economic divide within countries: a scorecard on the performance of regional development policies in reducing regional income disparities. World Development, v.31, n.8, p.1421-1441, 2003

SILVA, M. P. N.; PEROBELLI, F. S. Efeitos tecnológicos e estruturais nas emissões brasileiras de $\mathrm{CO}_{2}$ para o período 2000 a 2005: uma abordagem de análise de decomposição estrutural (SDA). Estudos Econômicos, São Paulo, v. 42, n. 2, p. 307-335, 2012.

SILVEIRA NETO, R. D. M.; AZZONI, C. R. Non-spatial government policies and regional income inequality in Brazil. Regional Studies, v. 45, n. 4, p. 453-461, 2011.

. Social policy as regional policy: market and nonmarket factors determining regional inequality. Journal of Regional Science, v. 52, n. 3, p. 433-450, 2012.

SKOLKA, J. Input-output structural decomposition analysis for Austria. In: KURZ, H. D.; DIETZENBACHER, E.; LAGER, C. (Ed.). Input-output analysis. Cheltenham, United Kingdom: Edward Elgar, 1989. v. III.

SOLOW, R. M. A contribution to the theory of economic growth. Quarterly Journal of Economics, v. 70, n. 1, p. 65-94, 1956.

SONIS, M.; HEWINGS, G. J. D. Fields of influence and extended input-output analysis: a theoretical account. In: DEWHURST, J. et al. (Eds.). Regional input-output modeling: new developments and interpretations. Avebury: Aldershot, 1991. p. 141-158.

SOUZA, N. J. Desenvolvimento polarizado e desequilíbrios regionais no Brasil. Revista Análise Econômica, Porto Alegre, v. 11, n. 19, p. 29-59, 1993.

WILLIAMSON, J. Regional inequality and the process of national development: a description of the patterns. Economic Development and Cultural Change, v. 13, n. 4, p. 1-84, 1965.

Recebido em: 22/05/2017. Aceito em: 31/03/2018. 